\title{
CARACTERÍSTICAS DAS TEMPESTADES COM RAIOS NO LITORAL DO PARANÁ
}

\author{
HEILMANN, Armando - heilmann@ufpr.br \\ Universidade Federal do Paraná / UFPR
}

\author{
FERNANDES, Caroline - carolfernadesoli@gmail.com \\ Universidade Federal do Paraná / UFPR
}

\author{
LEITE, Eduardo Alvim - alvim@simepar.br \\ Sistema Meteorológico do Paraná / SIMEPAR \\ PINHEIRO, Luciane Cristina - Iuciane@simepar.br \\ Sistema Meteorológico do Paraná / SIMEPAR
}

\begin{abstract}
RESUMO: Considerando um período de dezenove anos de dados de descargas atmosféricas monitorados pela Rede Nacional Integrada de Deteç̧ão de Descargas Atmosféricas - RINDAT, foi realizado um estudo das características das tempestades com raios no litoral do estado do Paraná. Com estes dados, que incluem informações sobre localização, data, pico de corrente e tipo de raios, foi possível a realização de análises quanto a distribuição espacial dos raios que ocorrem nesta região e sua relação com a topografia, o que mostrou as cidades com maior incidência de raios, particularmente em locais próximos da Serra do Mar, além de análises quanto a variação da quantidade de raios de acordo com as estações do ano e com períodos do dia, como também foi possível a realização do cálculo de uma estimativa do raio de atração das descargas atmosféricas que ocorreram no litoral paranaense durante o período estudado, utilizando valores de pico de corrente médio das descargas atmosféricas ao longo de todos os meses e de todos os anos. Em média, nas estações mais quentes $56,5 \%$ das descargas atmosféricas ocorreram sobre os municípios de Guaratuba e Guaraqueçaba. A orla das praias apresentou cerca de $7,7 \mathrm{raios} / \mathrm{km}^{2} / \mathrm{ano}$, sendo uma densidade de raios mais alta do que na cidade de Curitiba (com 7,01 raios $/ \mathrm{km}^{2} / \mathrm{ano}$ ). O horário predominante para as maiores frequências de raios são entre $18 \mathrm{~h}$ e $21 \mathrm{~h}$ (horário local), enquanto que em outonos e invernos, o ciclo diurno da atividade de raios não é bem definido. A estimativa do raio de atração, considerando a topografia da região, indica que cidades com maiores elevações também tiveram os menores valores médios de raio de atração, indicando uma maior probabilidade de incidência de raios nestes locais.
\end{abstract}

PALAVRAS-CHAVES: Tempestades; Descargas Atmosféricas; Topografia.

\section{LIGHTNING THUNDERSTORMS CHARACTERISTICS IN THE COAST OF PARANÁ}

ABSTRACT: Considering a period of nineteen years of atmospheric discharge data monitored by the Integrated National Atmospheric Discharge Detection Network RINDAT, a study of the characteristics of lightning storms on the coast of the state of Paraná was carried out. With this data, which includes information on location, date, current peak and lightning type, it was possible to analyze the spatial distribution of the lightning that occur in this region and its relationship with the topography, which showed the cities with the highest discharge near the Serra do Mar, as well as analyzes of the variation of the number of lightning according to the seasons and with periods of the day, as well as the calculation of an estimate of the radius of the attraction of the atmospheric discharges that occurred in the coast of Paraná during the studied period, using peak values of average current of the atmospheric discharges throughout all the months and of all the years. On average, in the hottest seasons $56.5 \%$ of the atmospheric discharges occurred on the cities of Guaratuba and Guaraqueçaba. The coast of the beaches presented about 7.7 flash/km2/year, being a lightning density higher than in the city of Curitiba (with 7.01 flash $/ \mathrm{km} 2 /$ year). The predominant time for the highest lightning 
frequencies is between $6 \mathrm{pm}$ and $9 \mathrm{pm}$ (local time), while in autumns and winters the daytime cycle of lightning activity is not well defined. The estimated radius of attraction, considering the topography of the region, indicates that cities with higher elevations also had the lowest average values of radius of attraction, indicating a greater probability of lightning incidence in these places.

KEYWORDS: Thunderstorms; Lightning; Topography.

\section{INTRODUÇÃO}

De acordo com GHRC (2015), o estudo e o mapeamento das descargas atmosféricas permitem: (a) detectar tempestades severas com antecedência; (b) estimar um valor de precipitação; (c) rastrear tempestades; (d) prever desastres na aviação; (e) avisar sobre riscos às companhias de energia e de combustível; (f) prever incêndios florestais; ( $g$ ) prever o desenvolvimento de ciclones; (h) entender a física do circuito elétrico global; e (i) entender a magnetosfera e a ionosfera, entre outros.

De acordo com Pinto Jr et al. (2000), a maior parte das descargas atmosféricas são provenientes de nuvens que possuem grande desenvolvimento vertical - nuvens do tipo Cumulus e Cumulunimbus. Estas nuvens se formam em condições específicas e o ar precisa estar suficientemente úmido e totalmente instável. $\mathrm{Na}$ região do litoral paranaense, estas nuvens de tempestade são geradas, na maioria das vezes, a partir da chegada de ar tropical quente e de ar polar frio (frentes frias) e sistemas convectivos de mesoescala durante as estações quentes (BARRY E CHORLEY, 2012).

A nuvem Cumulunimbus em seu estágio maduro apresenta regiões com partículas de cargas elétricas distintas. Estas partículas podem ser distribuídas na nuvem formando um dipolo ou um tripolo, sendo a região da base da nuvem ocupada por partículas com cargas negativas. Durante uma tempestade o solo adquire uma polaridade positiva, resultando num campo elétrico da ordem de 15 $\mathrm{kV} / \mathrm{m}$. Quando o campo elétrico da nuvem é suficientemente grande para romper a rigidez dielétrica do ar, o ar torna-se um condutor e o raio acontece (MACGORMAN, 1998).

A Serra do Mar causa grande influência no aumento da precipitação na região do litoral do Paraná, ocasionando chuva do tipo orográfica, que ocorre a partir da elevação forçada de parcelas úmidas de ar nas áreas de relevo acidentado (VANHONI E MENDONÇA, 2008). Segundo Barry e Chorley (2012) tal aumento da precipitação devido aos declives montanhosos é comum em latitudes médias, como também é uma característica dos subtrópicos a ocorrência de precipitação máxima abaixo dos picos montanhosos, com diminuição da intensidade em direção aos cumes. Além disso, uma barreira topográfica como a Serra do Mar pode produzir maior precipitação frontal, impedindo o avanço de massas de ar e provocando o retardamento do movimento de sistemas atmosféricos. Tais fatores que propiciam a formação de chuva orográfica também podem aumentar as condições de eletricidade dentro da nuvem, como será discutido adiante, sugerindo uma relação entre o aumento na precipitação e na quantidade de raios por efeito do relevo.

O ciclo diurno da atividade de raios é outra característica importante na análise de tempestades em uma região. Segundo Albrecht et al. (2016), a atividade de raios máxima relacionada ao continente tende a ocorrer no fim de tarde e a atividade máxima relacionada ao oceano tende a ocorrer nas horas 
noturnas. Esta tendência maior na ocorrência de raios sobre os oceanos, é devido à grande capacidade térmica e baixa porcentagem de albedo da água, que consegue armazenar o calor que recebe por mais tempo que o solo (REBOITA et al., 2012; BARRY E CHORLEY, 2012).

O que determina a capacidade térmica de corpos de diferentes naturezas é a densidade e o calor específico desses corpos. Tanto o calor específico quanto a capacidade térmica da água são maiores do que os da maioria dos corpos comuns, como a areia, por exemplo. Estas diferenças entre água e solo caracterizam a continentalidade. A continentalidade implica que uma superfície de terra se aquece e se resfria mais rápido do que o oceano e que as variações de temperatura são menores nas regiões litorâneas, que mesmo em posições geográficas distintas, podem apresentar médias térmicas aproximadas (BARRY E CHORLEY, 2012).

Na América do Sul, a chuva convectiva - conhecida como chuva de verão - aliada aos efeitos da continentalidade, atua como consequência do aumento da temperatura da superfície durante o dia, seguida de precipitação máxima no fim da tarde e início da noite (BURGESSER, 2014). Também é no final do dia que são observados os maiores registros de raios, desta forma, a chuva convectiva é mais comum nos meses mais quentes do ano e apresenta grande volume de água em um curto período de tempo, com uma incidência elevada de raios nas regiões onde ocorre.

Quanto à distribuição espacial dos raios, esta apresenta uma grande variação para diferentes locais em que é monitorada. Um estudo realizado por Bourscheidt et al. (2008) no Rio Grande do Sul sugere que o aumento na densidade de raios em uma região está relacionado com grandes variações de altitude, sendo a topografia uma grande influência na densidade de raios. Esta relação entre a variação de altitude e o aumento no número de raios pode ser comparada ao que ocorre na região do Lago de Maracaibo na Venezuela, local caracterizado por ser um grande vale - entre outros fatores - onde incidem mais raios em todo o mundo, sendo registrados até 297 dias de tempestade durante o ano (ALBRECHT, 2016).

Portanto, o propósito deste estudo é estabelecer algumas características referentes às tempestades com raios, analisando a distribuição espacial das tempestades com raios na região do litoral do Paraná e a severidade das tempestades que ocorreram para um determinado período, através da análise do pico de corrente sobre a região do litoral, estimado pela rede RINDAT.

\section{LOCALIZAÇÃO E CARACTERIZAÇÃO DA ÁREA DE ESTUDO}

O litoral do Paraná possui uma extensão de cerca de $100 \mathrm{~km}$ de comprimento, com área aproximada de $6600 \mathrm{~km}^{2}$ (VANHONI E MENDONÇA, 2008; ÂNGULO et al., 2006). Está localizado na costa da região Sul do Brasil, fazendo divisa com os estados de São Paulo ao norte e de Santa Catarina ao sul. É formado por sete municípios: Antonina, Guaraqueçaba, Guaratuba, Matinhos, Morretes, Paranaguá e Pontal do Paraná, sendo cinco deles banhados pelo Oceano Atlântico (Figura 1). Possui altitudes que variam de $0 \mathrm{~m}$ a aproximadamente $1.800 \mathrm{~m}$ na Serra do Mar. A Serra do Mar limita toda a planície litorânea paranaense, sendo atualmente a maior área preservada de 
Mata Atlântica em todo o Brasil (VANHONI E MENDONÇA, 2008; APA GUARAQUEÇABA, 1995).

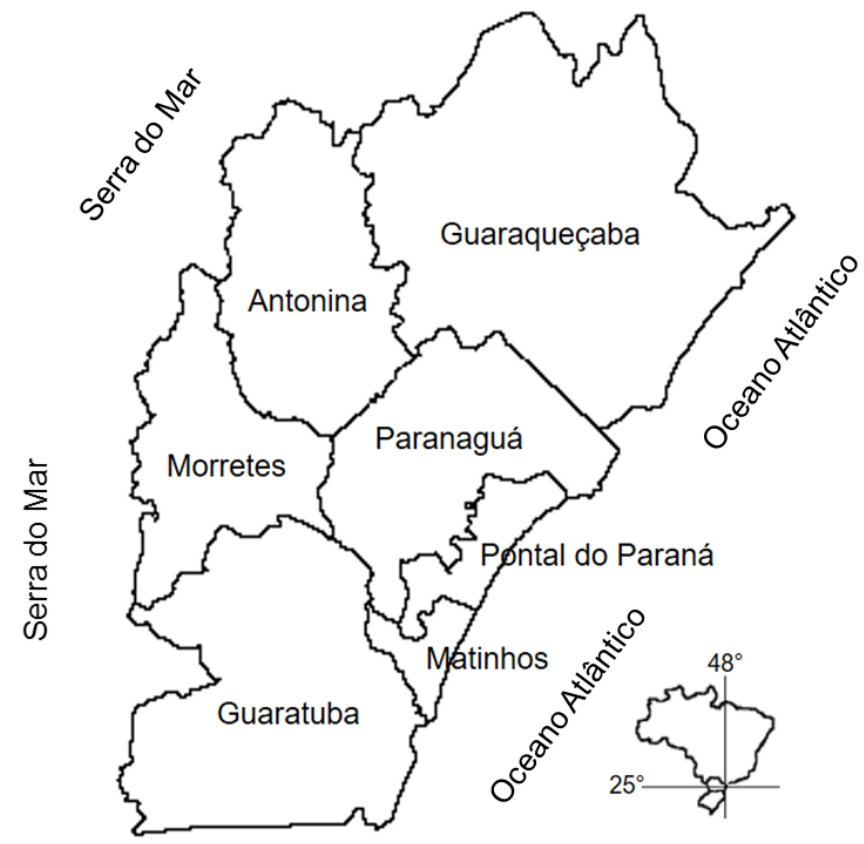

Figura 1 - Localização da área de estudo.

Segundo dados do Grupo de Eletricidade Atmosférica (ELAT-INPE, 2016), a densidade média anual de descargas atmosféricas nas cidades do litoral do Paraná é da ordem de 8,76 raios $/ \mathrm{km}^{2} /$ ano, sendo a cidade de Guaraqueçaba a que possui a maior média, com 10,17 raios/ km²/ano. Como comparação, a cidade de Curitiba apresenta a média de 7,01 raios/ km²/ano, e Foz do Iguaçu apresenta a média de 14,93 raios/ $\mathrm{km}^{2} /$ ano, possuindo a maior média paranaense. Porto Real/RJ, por exemplo apresenta uma média de 19,66 raios/ $\mathrm{km}^{2} /$ ano, sendo esta uma das cidades brasileira com maior densidade de raios. Tais diferenças ocorrem por uma combinação de diversos fatores como relevo, latitude, sistemas atmosféricos atuantes nas regiões e temperatura da superfície.

\section{FONTE E METODOLOGIA PARA TRATAMENTO DOS DADOS}

Foram utilizados dados de descargas atmosféricas que compreendem a categoria flash (flash é o agrupamento de vários strokes) registrados no período entre 1 de janeiro de 1997 à 31 de dezembro de 2015, pela RINDAT - Rede Integrada Nacional de Detecção de Descargas Atmosféricas (RINDAT, 2017), na qual o estado do Paraná faz parte através do SIMEPAR (SIMEPAR, 2017), que disponibilizou estes dados.

A incidência de descargas atmosféricas em muitas áreas é realizada por uma cobertura de uma rede de detecção, composta por sensores que registram as perturbações que esse fenômeno provoca no espectro de ondas eletromagnéticas, em especial em frequências de LF (Low Frequency - baixa frequência, entre 1 e $350 \mathrm{kHz}$ ). Essas perturbações possuem características muito específicas, o que permite realizar a separação de ocorrências de 
descargas atmosféricas de outros eventos que também possam causar perturbações nessa faixa do espectro. Nessa banda do espectro, a capacidade de detecção de descargas atmosféricas nuvem-solo pelos sensores da rede é maximizada, o que leva a uma maior confiabilidade e acurácia na detecção do fenômeno estudado.

Com as perturbações detectadas e identificadas pelos sensores da rede de detecção (sendo filtrados sinais espúrios), essas informações são enviadas dos sensores para uma central de processamento em tempo real por canais de comunicação (basicamente via internet), que irá receber e analisar todas as informações recebidas dos sensores. Essa análise leva em conta o timestamp ou a referência do instante que a perturbação foi detectada por um determinado sensor e/ou a direção da qual (em relação ao Norte verdadeiro) essa perturbação chegou até o sensor. Essas informações são utilizadas pelas técnicas Time of Arrival - TOA (somente o tempo de chegada da perturbação no sensor), Magnetic Direction Finding - MDF (ângulo de chegada da perturbação no sensor), e para a utilização conjunta entre as técnicas de TOA e MDF é dado o nome de IMPACT (IMProved Accuracy through Combined Technology).

Os raios são classificados como: nuvem-solo (que ocorre da nuvem para o solo), intra-nuvem (que ocorre dentro de uma mesma nuvem), nuvem-nuvem (que ocorre entre nuvens diferentes), nuvem-ar (que ocorre entre a nuvem e o ar) e solo-nuvem (que ocorre do solo para a nuvem). Dentre os raios que envolvem o solo, o tipo solo-nuvem é raro e chamado de ascendente (pois se origina no solo e termina próximo da nuvem) e acontece comumente em grandes elevações. O tipo nuvem-solo é mais comum e é uma descarga descendente (que se origina na nuvem e termina próximo do solo) (NACCARATO, 2001). Todas as descargas, porém, formam líderes ascendentes e descendentes.

Os raios podem ser classificados também quanto a polaridade da descarga elétrica: positivos, se gerados na região da nuvem com partículas de carga positiva, e negativos, se gerados na região onde as cargas negativas predominam. Dentre os raios do tipo nuvem-solo, os negativos são mais frequentes que os positivos, e dentre os raios intra-nuvem, os raios positivos são mais frequentes (RAKOV E UMAN, 2003; PINTO JR et al., 2000).

Os registros de raios positivos e negativos oferecem uma estimativa da intensidade do pico de corrente da descarga elétrica, monitorada e estimada pela RINDAT. Segundo Naccarato (2005), os sistemas de monitoramento perdem precisão e eficiência à medida que o pico de corrente dos raios diminui, e de acordo com Cummins (1998) e Orville (2002), as descargas de baixa intensidade monitoradas são geralmente do tipo intra-nuvem. Portanto, para análise do pico de corrente das descargas atmosféricas na região do litoral paranaense foram considerados os raios do tipo nuvem-solo, e foram excluídos os raios com valores de pico de corrente entre $10 \mathrm{kA}$ e -10kA, com o intuito de filtrar resultados equivocados de sua estimativa.

Esses dados então podem ser utilizados para a visualização em tempo real das ocorrências de descargas atmosféricas nas tempestades, bem como ser armazenadas em banco de dados para a realização de estudos e análises pretéritas. 
A RINDAT possui cerca de 40 sensores nas regiões Sul, Sudeste e Centro-Oeste do Brasil, além de 4 (quatro) centrais de processamento dos operadores dessa rede, localizadas em: Curitiba, Belo Horizonte, Rio de Janeiro e São José dos Campos. Os sinais dos sensores são transmitidos para as centrais de processamento através de canal de comunicação dedicado, e que posteriormente realizam a troca de informações dos sensores entre essas centrais, de forma que todas as centrais possam ter acesso a todos os sensores de forma simultânea, integrando a rede de detecção entre os operadores.

Os registros de raios são enviados às centrais de processamento, com o propósito de estimar, além da coordenada geográfica (latitude e longitude do raio) - com uma precisão de localização que, em geral, é inversamente proporcional a eficiência de detecção - e intensidade do pico de corrente (kA), outros parâmetros, como: polaridade, data, hora, nanosegundo, tempo de pico de corrente e elipse de incerteza da incidência do raio.

Os parâmetros analisados neste estudo foram: data (dia, mês, ano, hora, minuto e segundo), latitude, longitude, pico de corrente e classificação do raio (nuvem-solo e intra-nuvem). Estes dados foram tratados no software Excel por rotinas escritas, para fins de ilustrar as características das tempestades com raios no litoral do Paraná, por meio dos gráficos obtidos.

Os dados de altitude utilizados para a análise da topografia do litoral do Paraná foram obtidos pelo Shuttle Radar Topography Mission (SRTM) - NASA, com uma resolução de 1 arco-segundo ( 30 metros), acessados através do projeto TOPODATA (INPE, 2011), os quais foram tratados no software IDL (Interactive Data Language). Os dados obtidos pelo SRTM possuem informações acerca da elevação do terreno e são de uso exclusivamente científico.

A variação da quantidade anual de descargas atmosféricas por região será analisada considerando as variações anuais, que numa sequência temporal poderá ser observada através de linhas de tendência, para facilitar o entendimento quanto ao aumento de raios para os municípios do litoral paranaense.

\subsection{ANÁlISE DO CICLO DIURNO}

A análise do ciclo diurna de atividade dos raios, será feito considerando um estudo realizado por Easterly e Robinson (1985), que propuseram uma equação simples o qual indica se há um horário de pico na atividade de raios, ou dois picos, ou mesmo se não há picos regulares. A Equação (1) consiste em uma razão $V$ entre a amplitude da atividade máxima observada num período de 24 horas $(A)$ e a média da quantidade de raios neste período $\left(Q_{m}\right)$. Adaptando a equação de Easterly e Robinson para este estudo, temos:

$$
V=\frac{A}{Q_{m}}
$$

sendo $A$ é a amplitude da atividade máxima de raios em um dia, $Q_{m}$ é o número de raios médio em um período de 24 horas, e $V$ é a razão entre estes dois parâmetros.

Segundo Easterly e Robinson, é válida a seguinte análise: 
i. $\quad V<0.5$ indica falta de um pico bem definido ou dois picos na atividade diurna de raios;

ii. $\quad 0.5<V<1$ indica uma tendência com um claro pico na atividade diurna de raios;

iii. $\quad 1<V$ indica um ciclo diurno bem desenvolvido com um claro pico na atividade diurna de raios.

\subsection{ESTIMATIVA DO RAIO DE ATRAÇÃO ENTRE A DESCARGA ASCENDENTE E DESCENDENTE}

Com o propósito de estimar um raio de atração entre a descarga conectante ascendente com a descarga descendente, utilizamos a equação de Erikson (1979) e a norma 5419 da ABNT.

Dentre os sistemas de proteção contra descargas atmosféricas, o Modelo Eletrogeométrico (ou Método das Esferas Rolantes) é um dos mais eficientes. 0 conceito de raio de atração surge nessa teoria como sendo o comprimento da distância compreendida entre a ponta do líder descendente e a superfície onde se originou o líder ascendente. Este comprimento é utilizado como sendo o raio da esfera de proteção da estrutura atingida pela descarga atmosférica, segundo o Modelo Eletrogeométrico (VISACRO, 2004).

Aprimoramentos nesta teoria fizeram com que se passasse a considerar também a altura da estrutura no cálculo do Raio de atração, uma vez que estruturas mais altas necessitariam de um Raio de atração maior para que a proteção possa contemplar toda a estrutura. A equação de Erikson (1979) para o cálculo desta distância é a mais tradicional.

Como o objetivo deste estudo não é o de desenvolvimento de sistemas de proteção, o cálculo do raio de atração não levará em consideração as alturas dos locais de impacto das descargas atmosféricas, pois este parâmetro foi utilizado em uma análise topográfica posterior, relacionando a média dos Raios de Atração para cada cidade do litoral do Paraná (em função apenas do pico de corrente das descargas atmosféricas) com as respectivas topografias destas regiões.

Com os valores de pico de corrente dos raios de polaridade negativa nuvem-solo, registrados na região do litoral do Paraná, foi estimado o comprimento do raio de atração das descargas atmosféricas sobre a região. Este parâmetro pode ser obtido por meio de várias equações de diferentes autores, como Brown (1969), Armstrong (1968), Love (1973), Cooray (1997) ou pela norma 5419 da ABNT. Neste estudo, o raio de atração foi calculado de duas maneiras, através da Equação (2), de acordo com a norma 5419/2001 da ABNT utilizado para a proteção de estruturas contra descargas atmosféricas, e pela Equação (3), de Love (1973). Os resultados obtidos foram muito semelhantes para as duas expressões. 


$$
\begin{gathered}
R_{a}=2 \cdot I_{p}+30\left(1-e^{-I_{p}}\right), \\
R_{a}=10 \cdot I_{p}{ }^{0,65},
\end{gathered}
$$

sendo, $R_{a}$ é o comprimento do raio de atração das descargas atmosféricas [m] e $I_{p}$ é a intensidade do pico de corrente das descargas atmosféricas [kA].

\subsection{CLASSIfICAÇÃo SAZONAL dOS EVENTOS DE EL NIÑO, LA NIÑA E PERÍODOS NEUTROS}

Para classificar os eventos de El Niño e La Niña, além dos períodos neutros, foi consultado uma tabela com período histórico disponível no site da NOAA, que contém registros desde 1950. Nesta tabela, os períodos quentes (El Niño) correspondem aos períodos em que houve aumento maior ou igual a $0,5^{\circ} \mathrm{C}$ na Temperatura da Superfície do Mar (TSM) no Oceano Pacífico, enquanto os períodos frios ( $L a \mathrm{Niña)} \mathrm{correspondem} \mathrm{aos} \mathrm{períodos} \mathrm{em} \mathrm{que} \mathrm{a} \mathrm{TSM} \mathrm{esteve}$ pelo menos $0,5^{\circ} \mathrm{C}$ abaixo da média. Períodos em que a TSM variou menos do que $0,5^{\circ} \mathrm{C}$ acima ou abaixo da média são considerados neutros.

Disto, foram somadas as quantidades de raios a cada estação dos anos, fornecidos pelo registro dos sensores da RINDAT, comparado com os anos de El Niño e La Niña indicados na tabela da NOAA.

\section{RESULTADOS E DISCUSSÕES}

\subsection{DENSIDADE DE RAIOS NO LITORAL DO PARANÁ}

Foram detectadas 619.052 descargas atmosféricas do tipo nuvem-solo com intensidades maiores do que 10kA e menores do que -10kA, durante dezenove anos de monitoramento pela RINDAT (Janeiro de 1997 - Dezembro de 2015) no litoral do Paraná. Deste total, 227.175 ocorreram na cidade de Guaraqueçaba, o que representa $36,7 \%$ de todos os raios que ocorrem na região litorânea. A cidade de Guaratuba apresenta 19,8\% dos raios, seguida de Antonina, com 15,5\%. Em Paranaguá ocorreram 13,3\% dos raios, e em Morretes, 9,9\%. As cidades de Matinhos e Pontal do Paraná tiveram o menor número de ocorrências de raios, juntas somaram 4,7\% do total de descargas.

Embora Matinhos e Pontal do Paraná tenham apresentado os menores índices na ocorrência e registro de raios - bem como toda a orla das praias tenha mostrado menor densidade de raios - estas regiões possuem grande vulnerabilidade por se tratarem de locais onde se concentra o maior fluxo de turistas, que buscam atividades externas, principalmente durante o verão, ficando mais expostos ao risco de acidentes envolvendo descargas atmosféricas.

Segundo King (2015), ocorrem mais raios no continente do que nos oceanos porque o aquecimento diurno ocorre mais rapidamente na superfície do que nos oceanos. Quando ocorre a chegada de uma parcela de ar frio ao continente e esta parcela de ar frio encontra uma parcela de ar quente - aliada a grande umidade - o ar torna-se instável e intensificam-se os processos de formação e eletrificação das nuvens de tempestade. A interação entre massas de ar de diferentes temperaturas estimula a formação de tempestades e raios. 
Em geral, a quantidade anual de raios vem crescendo na região do litoral do Paraná. Um dos motivos hipotéticos pode estar relacionado ao aumento da temperatura, o que poderia resultar numa maior condição para os processos eletrostáticos dentro das nuvens de tempestade, outro motivo de um aumento no número de raios no litoral paranaense pode ser devido ao avanço da tecnologia das antenas e sensores de monitoramento de raios, que com o passar dos anos tem apresentado maior eficiência de detecção. Além de uma densidade de raios maior, a cidade de Guaraqueçaba também apresentou uma taxa maior de aumento na ocorrência de raios, ou seja, a quantidade anual de raios nesta região tende a crescer mais rápido do que nas cidades de Matinhos e Pontal do Paraná, por exemplo, que apresentaram as menores taxas de aumento (Figura 2).

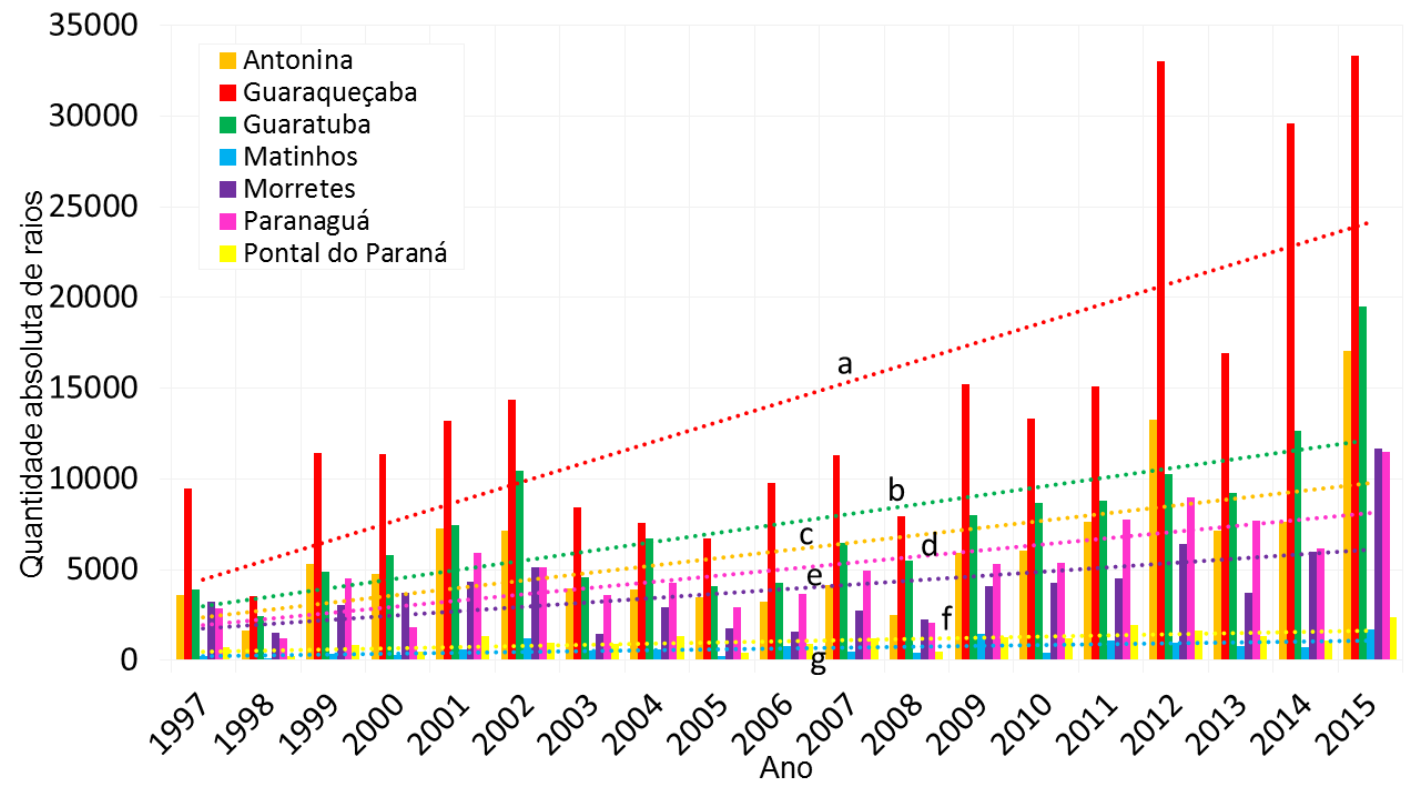

Figura 2 - Variação da quantidade anual de descargas atmosféricas que ocorreram nas sete cidades do litoral do Paraná entre 1997 e 2015. As linhas de tendência indicam: a: Guaraqueçaba, b: Guaratuba, c: Antonina, d: Paranaguá, e: Morretes, f: Pontal do Paraná e g: Matinhos. Fonte: RINDAT (2016).

Com os dados de topografia do litoral do Paraná foi possível criar um gráfico do relevo da região, com o objetivo de realizar uma análise descritiva espacial entre a densidade de raios e as elevações do terreno (Figura 3). 0 mapa de densidade de raios mostra que a distribuição das descargas não é uniforme em todo o litoral. Observou-se que os locais com maior concentração de raios são ladeados por elevações da Serra do Mar, especialmente Guaraqueçaba e Guaratuba, respectivamente com $210 \mathrm{flash} / \mathrm{km}^{2} / 19$ anos e 163 flashes $/ \mathrm{km}^{2} / 19$ anos. O número elevado de raios nas cidades de Guaraqueçaba, Guaratuba e Antonina, segundo estas análises, está relacionado às extensões desses municípios, bem como à distribuição da Serra do Mar.

A topografia contribui para uma intensificação dos processos de eletrização das nuvens de tempestade, especialmente sobre a região litorânea. 
As nuvens que encontram uma barreira topográfica são forçadas a subir, desenvolver-se verticalmente e se resfriar - fatores necessários para a evolução das condições eletrostáticas dentro da nuvem - o que resulta em um acréscimo no ciclo de vida da tempestade e numa sequência maior de raios por unidade de tempo, prolongando assim o decurso da ocorrência de raios sobre as regiões com estas características, como Guaraqueçaba e Guaratuba, diferente daquelas que estão ao nível do mar, livres da influência da topografia.

Schulz e Diendorfer (1999) encontraram uma relação entre a ocorrência de raios e grandes variações de altitude, e sugerem que a proximidade entre 0 centro de carga da nuvem e a superfície pode aumentar a possibilidade de ocorrência das descargas atmosféricas. De acordo com Wagtendonk e Cayan (2008), a topografia e a circulação diurna afetam a ocorrência espaço-temporal de raios, e a densidade de raios aumenta com a elevação topográfica de uma região.

Bourscheidt et al. (2007) observou no Rio Grande do Sul que o aumento da densidade de raios em uma região está relacionada à declividade do terreno e não, diretamente, à altura do morro, sendo as áreas com maior declividade também as que apresentam as maiores densidades de raios. Porém, este aumento não se estende até o topo do morro, onde há diminuição da ocorrência de raios. Isto explica o fato de que a maior densidade de raios não coincide com os setores mais elevados da Serra do Mar, mas sim em locais com alta declividade, conforme observado por esta pesquisa.

Rodrigues et al. (2010) propôs um mapa de densidade de raios nuvemsolo, usando dados da rede LLS (Lightning Location System) entre 2002 e 2007, em um estudo onde apresenta argumentos que indicam uma maior densidade de raios em regiões montanhosas do que em regiões planas, sugerindo, também, uma influência da topografia sobre a atividade de raios durante as tempestades.

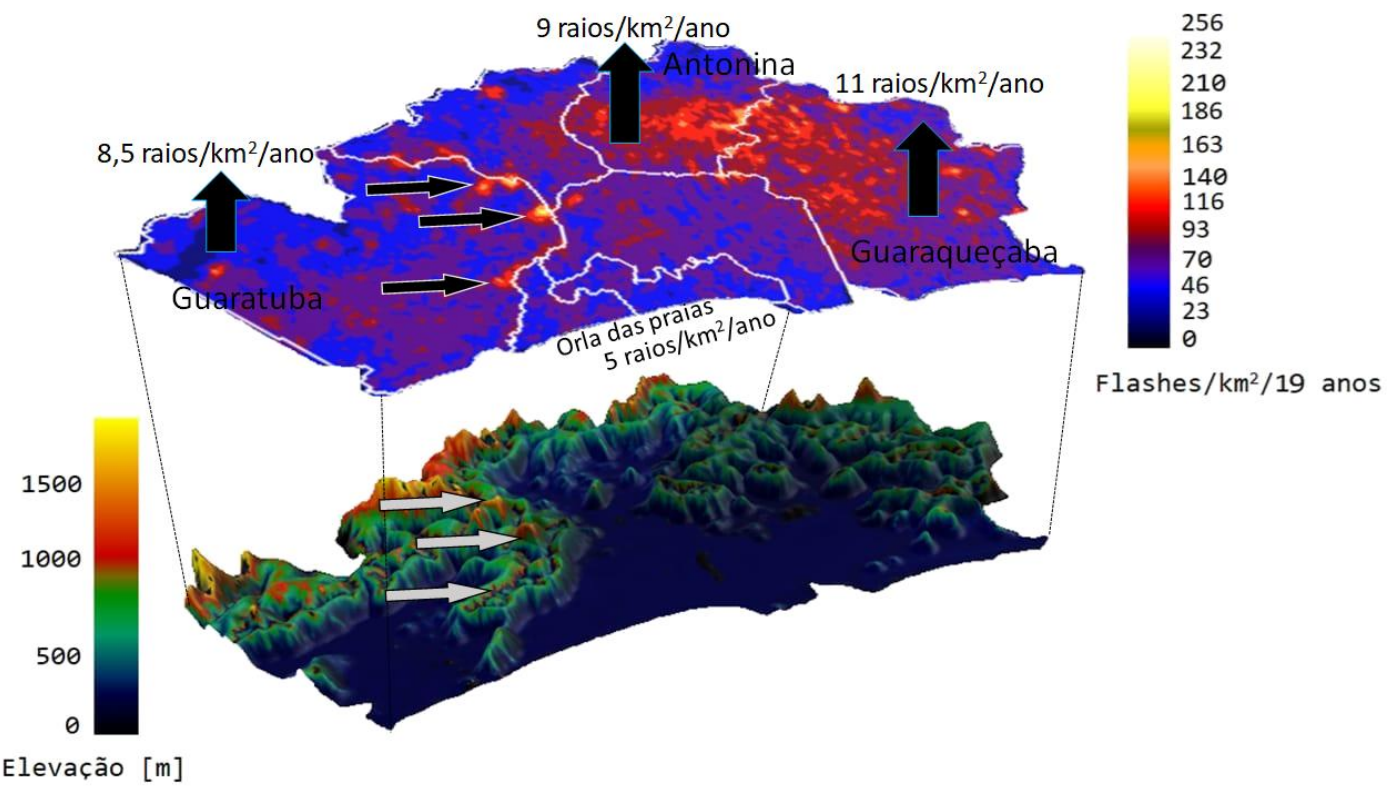

Figura 3 - Mapa da densidade de raios (acima) e da topografia (abaixo) do litoral do Paraná. Fonte: RINDAT (2016) e SRTM (2016). 
A grande variação de altitude que forma grandes vales entre a Serra do Mar na região do litoral do Paraná é uma característica que já foi observada por Vanhoni e Mendonça (2008). Estas regiões com elevações características também apresentaram maiores médias pluviométricas no estudo de Vanhoni e Mendonça (2008) sobre o litoral do Paraná, onde esse comportamento foi associado à presença de vales profundos nestas regiões, tornando estes locais favoráveis para a penetração de massas de ar úmido. A relação entre a precipitação e a quantidade de raios é mostrada na figura 4.

Tais variações mensais entre a quantidade de chuva e a quantidade de raios mostraram-se muito parecidas, com máximos nos meses quentes e mínimos nos meses mais frios do ano, estando de acordo com a literatura (ALBRECHT, 2016).

Desta forma, desconsiderando dados de radar meteorológico e a dinâmica de deslocamento das tempestades, e tomando somente os dados de raios e de temperatura da região do litoral do Paraná, tem-se que, principalmente a regiões de Guaraqueçaba e Guaratuba, oferecem às tempestades que se formam na região litorânea, uma condição de alta umidade e gradiente de temperatura baixo - típico de áreas litorâneas - que associado ao papel da Serra do Mar presente nesta região, contribui para um ciclo de vida mais prolongado das tempestades, e consequentemente uma frequência maior de raios, o que resulta numa densidade de raios sobre a região, igualmente maior.

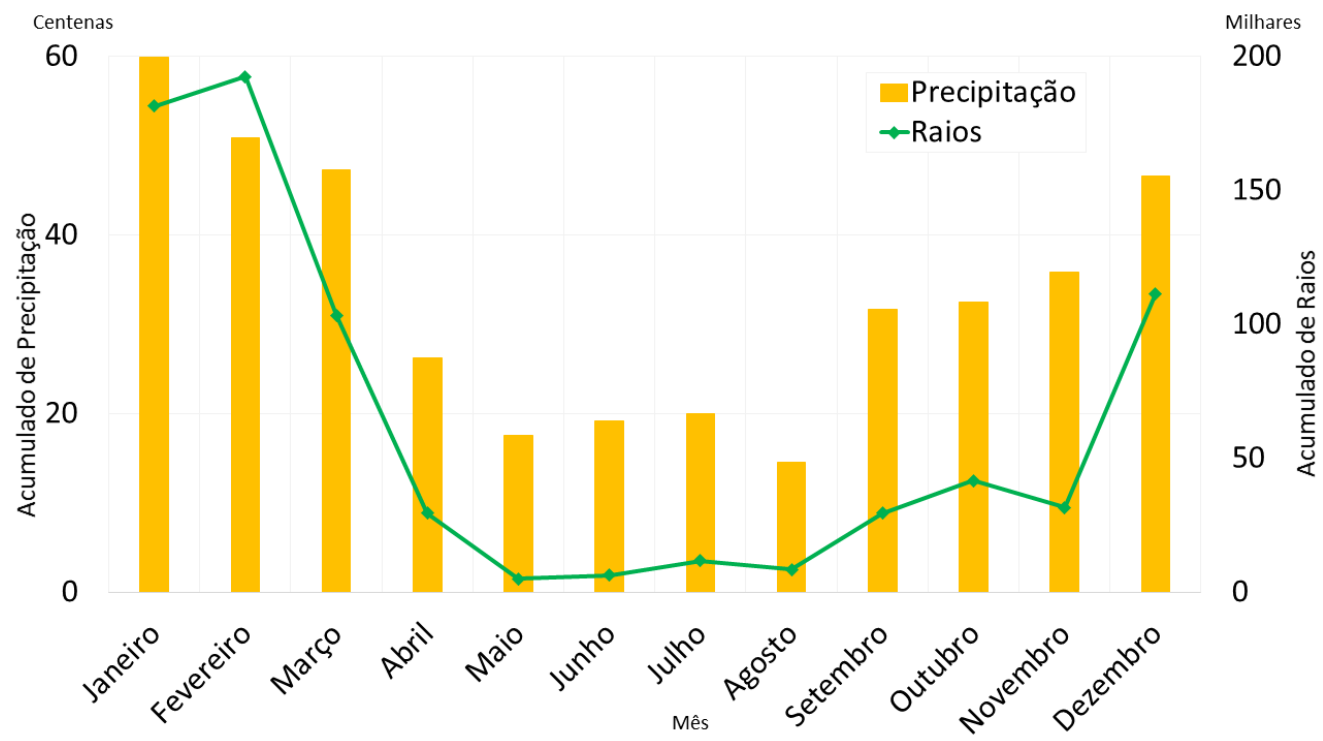

Figura 4 - Acumulado de precipitação média (em milímetros) e o acumulado médio de raios no decorrer de todos os meses, entre 1997 e 2015. Fonte de dados: BDMEP-INMET (2016) e SIMEPAR (2016).

\subsection{ANÁLISE DA QUANTIDADE ANUAL DE RAIOS POR ESTAÇõES DO ANO}

A análise entre a quantidade anual de raios e as estações do ano em que ocorreram esses raios apresentou a esperada predominância da incidência de raios nos verões, tanto positivos quanto negativos, seguido de primaveras, outonos e invernos - em ordem decrescente, respectivamente. 
Além disso, foi possível identificar visualmente o aumento na quantidade anual de raios em verões e primaveras, enquanto outonos e invernos apresentaram uma menor taxa de aumento na quantidade de raios entre os anos, durante o período considerado (Figura 5).

Esta variação mostra que o aumento na quantidade de raios na região do litoral do Paraná, durante o verão e a primavera, está relacionado ao aumento da temperatura da superfície nas estações quentes - intensificando o processo de evaporação da água - aliado ao deslocamento de sistemas convectivos típicos desta época do ano, como frentes frias.

A quantidade de raios em relação à ocorrência dos fenômenos El Niño e La Niña também foi analisada. Em períodos de ocorrência de El Niño foram registrados 247.605 raios, enquanto que períodos de La Niña somaram 184.255 raios, ou seja, foi observado cerca de 63 mil raios a mais em períodos de $\mathrm{El}$ Niño. A análise da figura 5 sugere que o aumento da quantidade de raios não está diretamente relacionado à ocorrência de um fenômeno ou outro, havendo uma variação cíclica de aumento e diminuição de raios, ora relacionada a períodos de El Niño e ora relacionados com períodos de La Niña, mas com um somatório final de raios maior para períodos de El Niño.

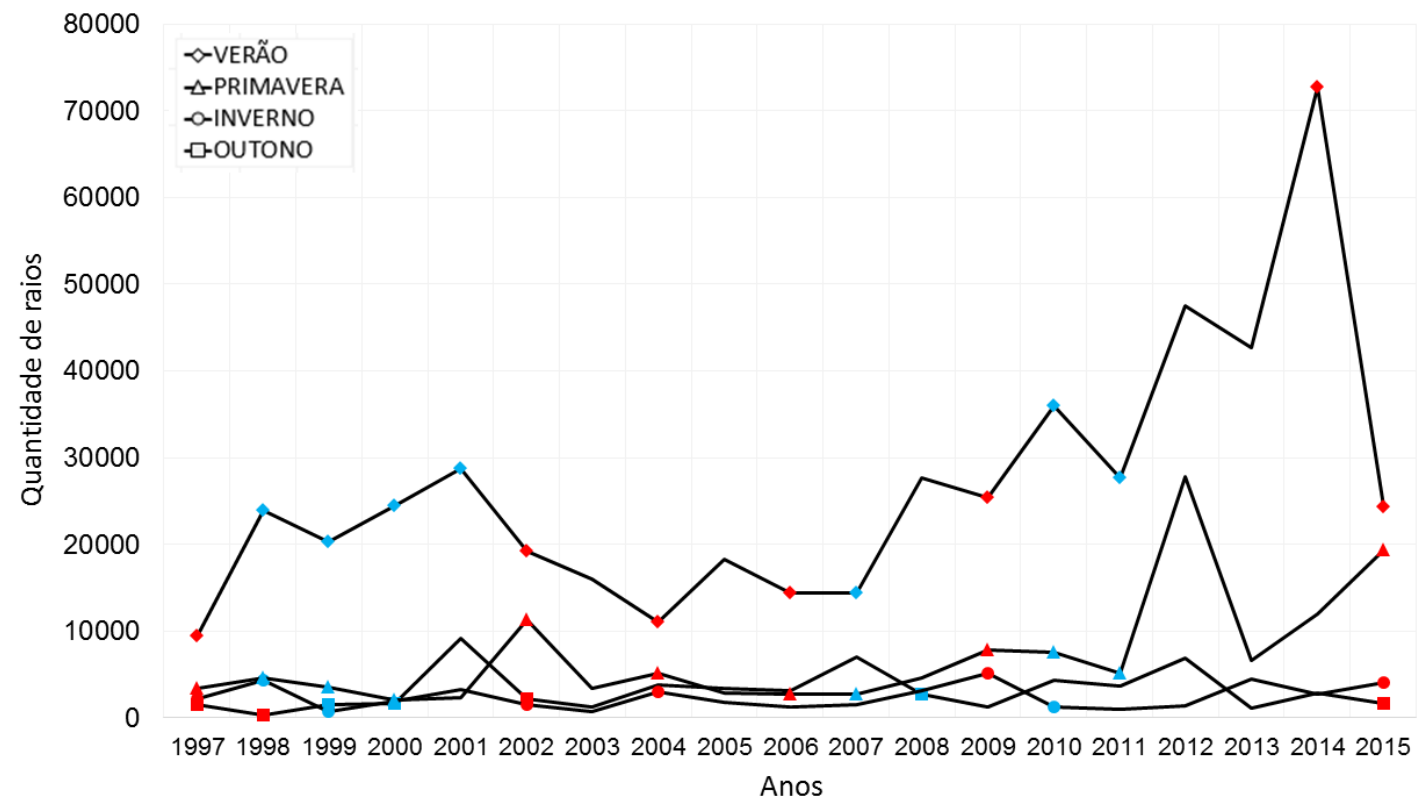

Figura 5 - Quantidade anual de raios por estações do ano entre 1997 e 2015. Os pontos em vermelho correspondem a períodos de ocorrência de El Niño, os pontos em azul correspondem a períodos de ocorrência de La Niña, e a ausência de pontos representa os períodos neutros. Fonte de dados: SIMEPAR (2016) e NOAA (2016).

Este resultado é coerente com a premissa de que eventos de El Niño causam mais precipitação e períodos de La Niña são caracterizados pela diminuição na precipitação no sul do Brasil (OLIVEIRA et al., 2009), logo, apresentam também um somatório maior de raios, já que a distribuição mensal de precipitação e de descargas atmosféricas é muito próxima, conforme observados na Figura 5. 


\subsection{CICLO DIURNO DA ATIVIDADE DE RAIOS}

A análise do período diurno (compreendido como sendo os períodos matutino e vespertino) que corresponde à atividade máxima de raios na região do litoral do Paraná, para o período entre 1997 e 2015 (Figura 6) mostrou um pico acentuado nos verões entre $15 \mathrm{~h}$ e $18 \mathrm{~h}$ (UTC), o que corresponde ao período entre $18 \mathrm{~h}$ e $21 \mathrm{~h}$ horário local. Durante as primaveras e outonos também mostraram predominância na ocorrência de raios no fim de tarde e início da noite. Porém, nos invernos verifica-se uma irregularidade quanto aos horários de maior ocorrência de raios.

A atividade máxima de raios é predominante em fins de tarde e inícios da noite, entre $18 \mathrm{~h}$ e $21 \mathrm{~h}$ no horário local, principalmente nas estações mais quentes, está relacionada ao aquecimento diurno da superfície, que aumenta gradualmente a evaporação formando umidade e produzindo precipitação e ocorrência máxima de raios próximo das horas noturnas, conforme também observado por Burgesser et al. (2014). valores:

Usando a equação (1) de definição do ciclo diurno, obtemos os seguintes

Para o outono, $V_{\text {out }}=0.235$, que corresponde ao item i. Para o inverno, $V_{i n v}=$ 0.131 , que corresponde ao item i. Para a primavera, $V_{\text {prim }}=0.65$ que corresponde ao item ii. Para o verão, $V_{\text {ver }}=1.083$ que corresponde ao item iii.

Estes resultados indicam que a variação do ciclo diurno da atividade de raios entre as estações do ano no período considerado estão de acordo com a equação de Easterly e Robinson, sugerindo que este modelo de estimativa da variação do ciclo horário das tempestades com raios pode ser aplicada para a região do litoral do Paraná.
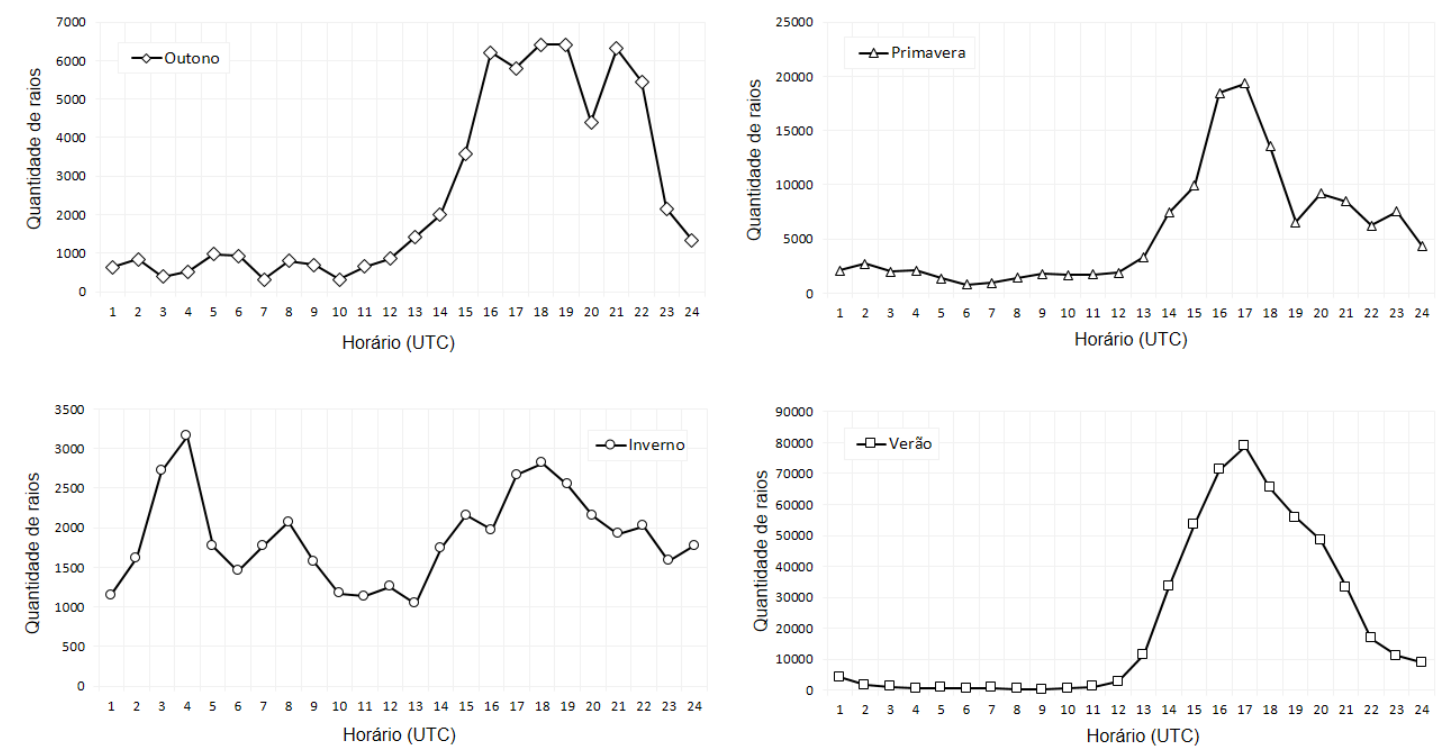

Figura 6 - Análise do ciclo diurno da atividade de raios. Fonte: RINDAT (2016). 


\subsection{ANÁLISE DO PICO DE CORRENTE DAS DESCARGAS ATMOSFÉRICAS}

Do total de 619.052 raios nuvem-solo monitorados sobre o litoral do Paraná no período considerado, $518.073(83,7 \%)$ possuem pico de corrente negativo e 100.979 (16,3\%) apresentam pico de corrente positivo. Em um estudo feito por Visacro et al. (2004) para 79 raios com incidência sobre uma torre de $1.430 \mathrm{~m}$ (Morro do Cachimbo - MG), destes, $81 \%$ foram de polaridade negativa e $16,5 \%$ de polaridade positiva, porcentagens estas muito próximas das encontradas a partir dos dados da RINDAT sobre o litoral do Paraná, em dezenove anos.

Os valores de pico de corrente médio apresentaram pouca variabilidade entre as sete cidades do litoral do Paraná durante o período monitorado, ou seja, a intensidade média das descargas é semelhante em todas as regiões. Estes valores apresentaram também uma ligeira tendência de queda no decorrer dos anos, para todas as cidades (Figura 7).

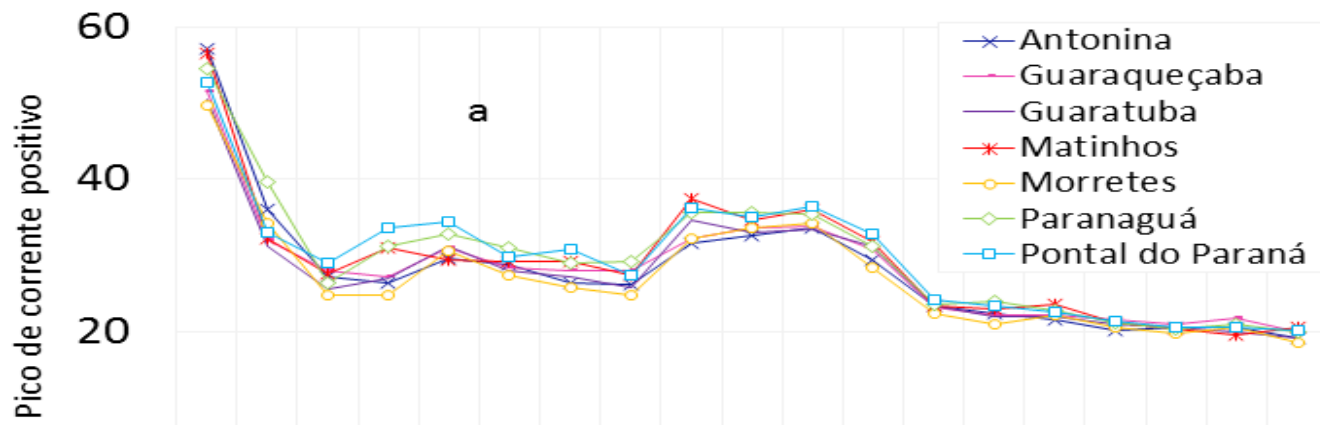

0

1997199920012003200520072009201120132015
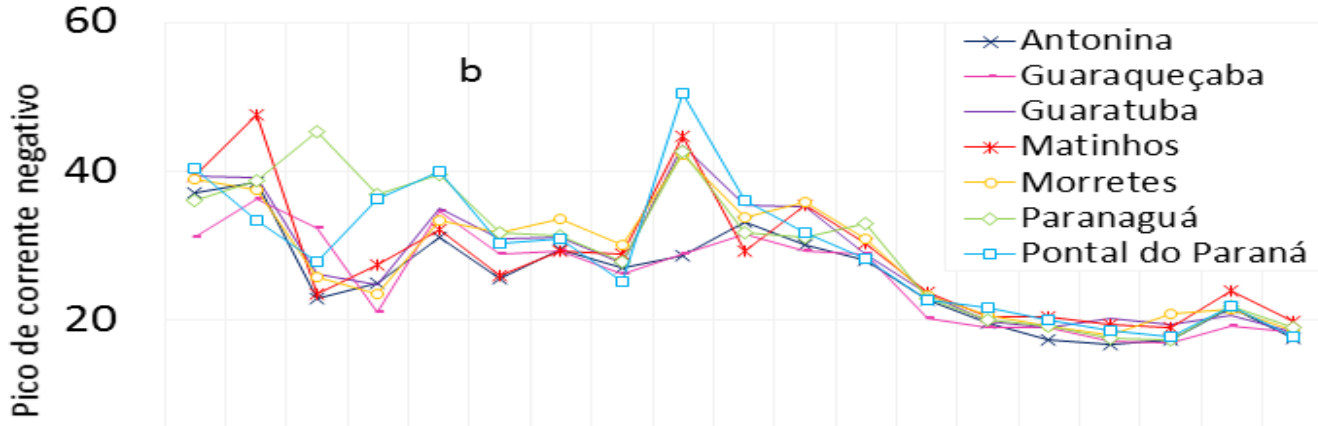

0

1997199920012003200520072009201120132015

Figura 7 - Comparação do pico de corrente anual médio entre as cidades do litoral do Paraná. Fonte: RINDAT (2016).

\subsection{ESTIMATIVA DO RAIO DE ATRAÇÃO}

Esta análise mostrou que as cidades de Morretes, Antonina, Guaratuba e Guaraqueçaba, municípios próximos da Serra do Mar, apresentam um comprimento R_a menor do que as cidades de Pontal do Paraná, Paranaguá e Matinhos, quase que inteiramente costeiras e ao nível do mar. Isso se deve ao 
fato de que quanto maior a altitude, menor é a distância entre a extremidade do líder descendente com a do líder ascendente. Em outras palavras, isto significa que as regiões que apresentam morros são mais propensas à incidência de raios, devido a condição facilitada para o acoplamento entre os canais de ambos os líderes. Isto corrobora como o que foi observado na análise da densidade de raios (Figura 3). A Figura 8a indica o comprimento $\mathrm{R}$ a e a Figura 8b apresenta a relação encontrada entre o R_a calculado a partir das equações (1) e (2), e as cidades do litoral paranaense.

A cidade de Morretes, que apresentou a menor distância Ra é também a cidade que apresenta os morros com maiores elevações da Serra do Mar, como o Pico Paraná com 1.922 m, mas não necessariamente é a cidade com maior densidade de raios, conforme seção 4. A cidade de Pontal do Paraná, pelo contrário, está quase que inteiramente ao nível do mar e não apresenta grandes elevações, e apresentou valores Ra maiores.

Apesar de apresentar pouca variação entre as cidades do litoral do Paraná, como visto anteriormente, os valores de pico de corrente utilizados como parâmetro para a obtenção da estimativa de Ra foram suficientes para indicar uma relação de origem topográfico quanto a formação dos raios, com relação a capacidade de acoplamento entre seus líderes ascendentes e descendentes, sobre a região do litoral paranaense.

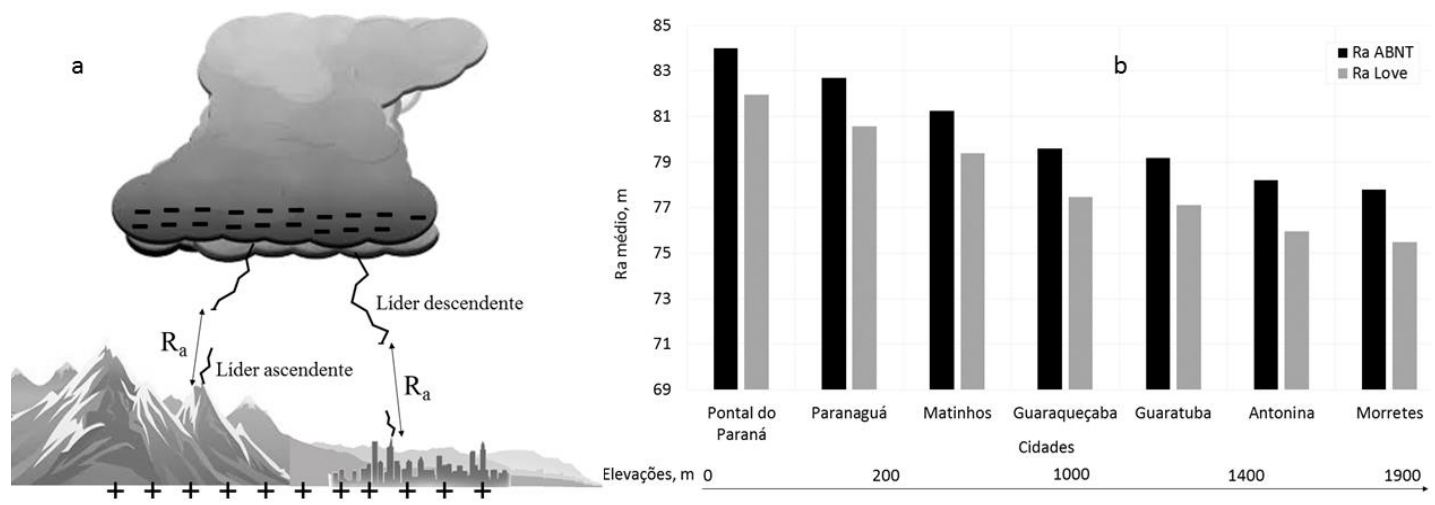

Figura 8 - a Comprimento da medida do raio de atração das descargas atmosféricas. b Raio de atração médio entre as cidades do litoral do Paraná, comparando com suas elevações.

\section{CONCLUSÃO}

Diante do propósito de analisar a distribuição das descargas atmosféricas e dos parâmetros que estão associados às características das tempestades na região do litoral do Paraná, é possível afirmar que o conjunto de dados dos 19 anos de registro de raios confere, ao objetivo deste trabalho, uma interpretação muito importante no entendimento de como as tempestades com raios se comportam, tanto para diferentes estações do ano, quanto para diferentes fases do ENOS.

A partir deste estudo foi possível concluir que, em geral, a quantidade anual de raios vem aumentando na região do litoral do Paraná. Os municípios de 
Guaratuba e Guaraqueçaba mostraram uma maior ocorrência de descargas atmosféricas, somando juntas $56,5 \%$ do total de descargas atmosféricas na região, e apresentaram uma maior taxa de aumento anual de descargas. A maior ocorrência de raios é observada nas estações quentes, primavera e verão, como também as estações quentes apresentam uma maior taxa de aumento anual de raios.

A orla das praias apresentou uma menor ocorrência de descargas atmosféricas, porém, por ser esta uma região que atrai mais turistas, por permitir mais atividades ao ar livre, deixa a população mais exposta ao risco de incidência por raios. Embora a incidência de raios seja menor nesses locais, da ordem de 7,7 raios $/ \mathrm{km} 2 /$ ano, ainda assim é mais alta do que na cidade de Curitiba (com 7,01 raios/km2/ano).

A análise da severidade das tempestades sobre o litoral do Paraná mostrou que as estações mais quentes produzem mais raios. O horário de maior ocorrência das atividades com raios foi observado entre $18 \mathrm{~h}$ e $21 \mathrm{~h}$ (horário local) em primaveras e verões, que correspondem aos horários de maior vulnerabilidade da população ao risco de acidentes envolvendo raios. Já em outonos e invernos, o ciclo diurno da atividade de raios não é bem definido.

Além disso, os valores de pico de corrente também foram utilizados para criar uma estimativa do raio de atração, considerando a topografia da região, indicou que as cidades com presença de maiores elevações, foram também as cidades que tiveram os menores valores médios de raio de atração, indicando uma maior probabilidade de incidência de raios nestes locais.

\section{AGRADECIMENTOS}

Os autores agradecem ao Sistema Meteorológico do Paraná - SIMEPAR pela contribuição com os dados fornecidos e pela colaboração na análise climatológica sobre a região.

\section{REFERÊNCIAS BIBLIOGRÁFICAS}

ALBRECHT, R.; GOODMAN, S.; BUECHLER, D.; BLAKESLEE, R.; CHRISTIAN, H. Where are the lightning hotspots on Earth? Bulletin of the American Meteorological Society, v. 97, p. 2051-2068, 2016.

ÂNGULO, R. J.; SOARES, C. R.; MARONE, E.; SOUZA, M. C.; ODRESKI, L. L. R.; NOERNBERG, M. A. Erosão e Progradação do Litoral Brasileiro - Paraná. Brasília: MMA, 2006. 476 p.

ARMSTRONG, H. R.; WHITEHEAD, E. R. Field and Analytical Studies of Transmission Line Shielding. IEEE Transactions on Power Apparatus and Systems, v. 87, p. 270-281, 1968.

Associação Brasileira de Normas Técnicas (ABNT). NBR 5419: Proteção de estruturas contra descargas atmosféricas. Rio de Janeiro, p. 28, 2005.

BARRY, R. G.; CHORLEY, R. J. Atmosfera, Tempo e Clima. 9a. ed. Porto Alegre: Bookman, 2012. 528 p. 
BOURSCHEIDT, V. Estudo da relação entre relâmpagos, relevo, temperatura de superfície e tipos de solo no Rio Grande do Sul. 2008. 126p. Dissertação de Mestrado em Geofísica Espacial, Instituto Nacional de Pesquisas Espaciais, São José dos Campos, 2008.

BOURSCHEIDT, V.; JUNIOR, O. P.; PINTO, I. R. C. A.; NACCARATO, K. P. The influence of topography on cloud-to-ground lightning characteristics at South Brazil. Atmospheric Research, v. 91, p. 508-513, 2008.

BROWN, G. W.; WHITEHEAD, E. R. Field and Analytical Studies of Transmission Line Shielding - Part II. IEEE Transactions on Power Apparatus and Systems, v. 88, p. 617-626, 1969.

BURGESSER, R. E.; CASTELlANO, N. E.; NICORA, M. G.; AVILA, E. E. Diurnal cycle of lightning activity over continental regions. XV International Conference on Atmospheric Electricity. Oklahoma, USA, 2014.

CANE, M. A. The evolution of El Niño, past and future. Earth Planet Science Letters, v. 164, p. 1-10, 2005.

COORAY, V. A model for negative first return strokes in negative lightning flashes. Physica Scripta, v. 55, p. 119-128, 1997.

CUMMINS, K. L.; KINDER, E. P.; MALONE, M. D. The US national lightning detection network and applications of cloud-to-ground lightning data by electric power utilities. IEEE Transactions on Electromagnetic Compatibility, v. 40, p. 465-480, 1998.

EASTERLY, D. R.; ROBINSON, P. J. The diurnal variation of thunderstorm activity in the United States. Journal of Climate and Applied Meteorology, v. 24, p. 1048-1058, 1985.

ERIKSSON, A. J. The lightning ground flash: an engineering study. 1979. 400p. PhD thesis, Faculty of Engineering, University of Natal, Pretoria, South Africa, 1979.

GHRC (Global Hydrology Resource Center). Lightning and Atmospheric Electricity Research. 2015. Disponível em:

<https://lightning.nsstc.nasa.gov/primer/primer4.html> Acesso em 8 de outubro de 2016.

INPE (Instituto de Pesquisas Espaciais). Projeto TOPODATA. 2011. Disponível em: <http://www.dsr.inpe.br/topodata/>. Acesso em 22 de julho de 2014.

INPE (Instituto de Pesquisas Espaciais). Grupo de Eletricidade Atmosférica (ELAT). Disponível em: <http://www.inpe.br/webelat/homepage/> Acesso em 12 de setembro de 2016.

INSTITUTO AMBIENTAL DO PARANÁ. Secretaria de Estado do Meio Ambiente e Recursos Hídricos. Plano de Gestão Ambiental - Área de Proteção Ambiental de Guaraqueçaba. Curitiba: IAP 1995.

KING, H. World Lightning Map. 2015. Disponível em:

$<$ http://geology.com/articles/lightning-map.shtml>. Acesso em 8 de outubro de 2016. 
LOVE, E. R. Improvements on Lightning Stroke Modeling and Applications to Design of EHV and UHV Transmission Lines. MSc Thesis, University of Colorado, Denver, 1973.

MARONE, E.; ÂNGULO, R. J.; MARTINS, G. J.; PEREIRA, J.; NETTO, B. Sistemas frontais em duas praias do litoral paranaense. Boletim Paranaense de Geociências, n. 60-61, p. 65-74, 2007.

MACGORMAN, D. R.; RUST, W. D. The Electrical Nature of Storms. New York: Oxford University Press, 1998. 422p.

NACCARATO, K. P.; PINTO JR., O.; PINTO, I. R. C. A. Cloud-to-ground lightning flash climatology of State of São Paulo: 1999-2004. VII International Symposium on Lightning Protection (SIPDA), p. 120-125, 2005.

NACCARATO, K. P.; Estudo de relâmpagos no Brasil com base na análise de desempenho do Sistema de Localização de Tempestades. 2001. 165p. Dissertação de Mestrado em Geofísica Espacial, Instituto Nacional de Pesquisas Espaciais, São José dos Campos, 2001.

OLIVEIRA, G. S.; SILVA, N. F.; HENRIQUES, R. Mudanças climáticas - Coleção Explorando o ensino, v. 13. Brasília: MEC, SEB; MCT; AEB, 2009. 348p.

ORVILLE R. E.; HUFFINES G.; BURROWS W. R.; HOLLE R. L.; CUMMINS K. L. The North American Lightning Detection Network (NALDN) - First Result: 19982000. Monthly Weather Review, v. 130, p. 2098-2109, 2002.

PINTO JR., O.; PINTO, I. R. C. A. Tempestades e relâmpagos no Brasil. 185p. 2000. Instituto Nacional de Pesquisas Espaciais, São José dos Campos, 2000.

RAKOV V. A.; UMAN M. A. Lightning: Physics and Effects. Cambridge University Press, 2003. 687p.

REBOITA, M. S.; KRUSCHE, N.; AMBRIZZI, T.; ROCHA, R. P. Entendendo o Tempo e o Clima na América do Sul. Terra e Didática, v. 8, p. 34-50, 2012.

RODRIGUES, R. B.; MENDES, V. M. F.; CATALÃO, J. P. S. Lightning data observed with lightning location system in Portugal. IEEE Transactions on Power Delivery, v. 25, p. 870-875, 2010.

SCHULZ, W.; DIENDORFER, G. Lightning characteristics as a function of altitude evaluated from lightning location network data. International Conference on Lightning and Static Electricity (ICOLSE). Toulouse, France, 1999.

VANHONI, F; MENDONÇA, F. O Clima do Litoral do Estado do Paraná. Revista Brasileira de Climatologia, v. 12, p. 49-63, 2008.

VISACRO, S.; SOARES JR., A.; SCHROEDER, M. A.; CHERCHIGLIA, L. C. L.; SOUSA, V. J. Statistical analysis of lightning current parameters: Measurements at Morro do Cachimbo Station. Journal of Geophysical Research, v. 109, 2004.

WAGTENDONK, JAN W. VAN; CAYAN, D. R. Temporal and Spatial distribution of lightning Strikes in California in relation to large-scale Weather patterns. Fire Ecology, vol. 4, n. 1, p. 34-56, 2008. 